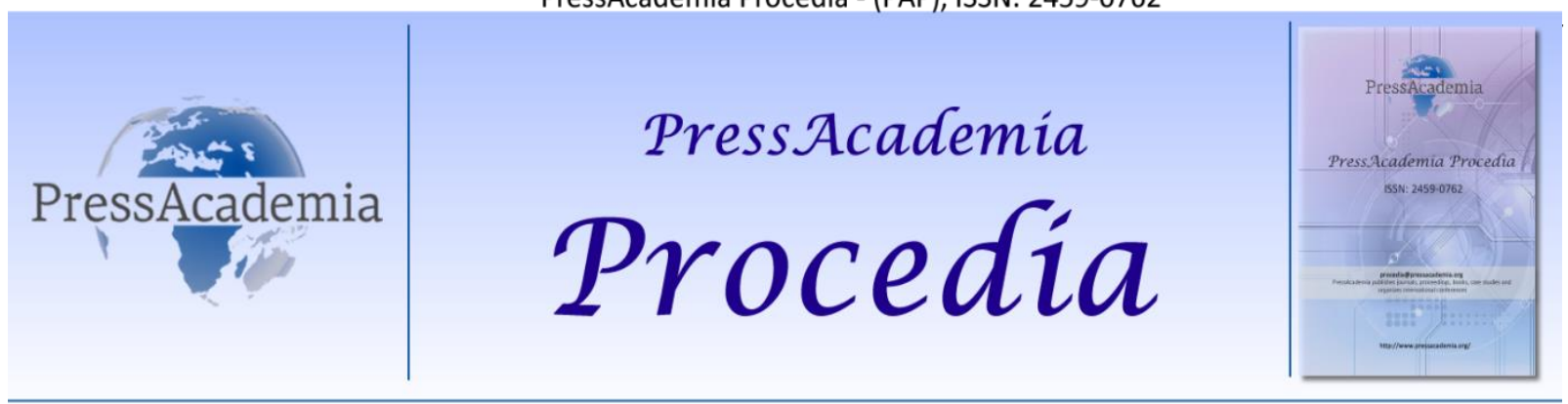

2nd World Conference on Technology, Innovation and Entrepreneurship

May 12-14, 2017, Istanbul, Turkey. Edited by Sefer Şener

\title{
SOLVOTHERMAL SYNTHESIS AND BIOLOGICAL ACTIVITY OF NI-DOPED ZINC OXIDE NANOPARTICLES
}

\author{
DOI: 10.17261/Pressacademia.2017.595 \\ PAP-WCTIE-V.5-2017(33)-p.240-246
}

Sammia Shahid ${ }^{1} *$, Muhammad Mudassar Sher ${ }^{2}$

${ }^{1}$ Department of Chemistry, School of Science, University of Management \& Technology, Lahore-, Pakistan. sammia.shahid@umt.edu.pk

${ }^{2}$ Department of Chemistry, School of Science, University of Management \& Technology, Lahore-, Pakistan. mudassarsher786@gmail.com

\begin{abstract}
Metal oxide nanoparticles are potential candidate for making future antimicrobials. Increased interest is due to change in fundamental properties at nanoscale. Ni-doped zinc oxide nano-particles were prepared for pharmacological studies. Co-precipitation and solvo-thermal methods were employed which yielded Ni-doped zinc oxide nano-particles and un-doped zinc oxide nano-particles were synthesized via solvo-thermal method. All prepared nano-particles were characterized using X-ray diffraction studies whereas doping was confirmed by Energy Dispersive X-ray analysis. Shape and morphology of these nano-particles was assessed using Scanning Electron Microscopy. The synthesized nano-particles have shown antibacterial activity against both Gram-negative and Gram-positive bacteria designating these nano-particles as future broad spectrum antibacterial. The optical properties were also studied by measuring the energy band gap and were found $1.50 \mathrm{eV}$ for un-doped zinc oxide nano-particles, and it decreases to $1.47 \mathrm{eV}$ for $\mathrm{Ni}$-doped zinc oxide. Ni-doped zinc oxide nanoparticles were proved to be active future pharmaceutical and biomedical agents.
\end{abstract}

Keywords: Antibacterials, morphology, nanoparticles, energy dispersive X-ray, doping

\section{INTRODUCTION}

The particles, rods and systems having one or more dimensions in nanometer scale range are the possible smallest things that we can produce up till now (Liewhiran et al., 2006). The particles having size ranges from 1-100 nanometers are called as nano-particles. These particles are unique in their properties and they also find applications in various fields (Hewakuruppu et al., 2013). For instance nano-particles are being used electronics, energy production, biosciences and medicines (B nuzea et al., 2007).

Similarly nano-particles of metallic origin found applications in electronics, medicine and in many other industries (Sahoo et al., 2007). Metals have been used as raw materials to produce distinct nanostructures such as nano-rods, nano-nails, nanowires, nano-tubes and nano-belts besides nano-particles alone (Hsu et al., 2006). Oxides of some metals are also in use for their ease in fabrication to nano materials. For example Zinc oxide has been used to produce nano-particles, nano-sheets, nano-tubes, nano-wires, nano-belts and nano-rings, etc. (Hua et al., 2007).

Bio-inspired productions of Zinc oxide nano-particles were achieved using environmentally along with eco-friendly accepted systems (Cai et al., 2013). Different chemical methods have been used to prepare Zinc oxide nano-particles by using different materials of interest. The majority of the Zinc oxide crystals were synthesized by using traditional high temperature (solid state) method but in this method it is difficult to organize the particle properties along with energy consumption. Zinc oxide nano-particles can also be prepared on a big scale at low cost by using simple solution based methods, such as chemical precipitation, hydrothermal reaction and sol-gel synthesis (Zhong et al., 1996).

Zinc oxide nano-particles are most favored due to its applications such as transparent conducting films, gas sensor, piezoelectric transducers as well as photo catalytic activity (Zhang et al., 2009). Zinc oxide nano-particles are used in different commercial products for instance in cosmetics, sunscreens and for anti-bacterial activity (Xu et al., 2010). Other nanostructures of Zinc oxide are also found applications in optoelectronics, transducers, sensors, and biomedical sciences 
(Wang et al., 2004). Doping of Zinc oxide nano-particles with other metals such as $\mathrm{Ni}, \mathrm{Ga}$, In, Sn, Al, Y, and Sc, are also in practice to improve properties Zinc oxide nano-particles (Moriga et al., 2004). Nickle recognizes as one of the most proficient doping element to advance as well as tune the electrical and optical properties of Zinc oxide nano-particles. The main emission peak of Zinc oxide is found as a sturdy ultraviolet (UV) emission at $380 \mathrm{~nm}$. (Al-Harbi et al., 2011).The interest to study optical properties of Zinc oxide nano-particles doped with Ni must provoke to develop an understanding of the material response to doping. There are numerous reports presented in the literature on optical properties Zinc oxide nano-particles doped with transition metals (Yan et al., 2003).

The nano-particles and nano-rods have been prepared by various groups using different methods such as sol-gel process, spray paralyses, laser molecular beam epitaxy, metal organic chemical vapor deposition, and pulsed laser deposition. Among these, the solvo-thermal methods have surpassed the others due to possessing better homogeneity, being low-cost, and being simple (Wani et al., 2007). The synthesized nano-particles by above method have high surface area as compared to other methods, low in cost and ease of synthesis. These synthesized nano-particles found applications as antibacterial and antimicrobial agent (Hadjipanayis et al., 1994). The characterization techniques have been used for nano-particles such as Scanning electron microscopy (SEM), Transmission electron microscopy (TEM), X-ray diffraction (XRD), Photoluminescence (PL), Energy Dispersive X-Ray Analysis (EDX), UV-visible Absorption Spectroscopy and Superconducting Quantum Interference Device (SQUID) (Ananda et al., 2014).These techniques are developed due to the recent research in nano-science and nanotechnology is considered most important as well as most growing technologies (Khatoon et al., 2012).

Diseases have been the threat to human being since ancient times and these were mostly caused pathogenic microbes. Bacteria as part of pathogenic microbes' community are responsible spreading different minute and chronic diseases. Although different antibacterials are available in the market but research is going on to develop various chemical agents known as antibacterials to cope this threat in best way. The production of nano-particles their property to kill bacteria has been proving as alternative to conventional medicines. Antibacterial activity of different types of $s$ Therefore it is of great importance to know and to understand antimicrobial effects synthesized nano-particles have been reported in the literature. The effect of doping to nano-particles is the focus of this study.

\section{DATA AND METHODOLOGY}

The chemicals used were obtained from Merck. The chemicals used were $\mathrm{Zn}(\mathrm{CH} 3 \mathrm{COO})_{2} 2 \mathrm{H}_{2} \mathrm{O}$ (zinc acetate dehydrate) $\mathrm{NiCl}_{2}$ $6 \mathrm{H}_{2} \mathrm{O}$ (nickel chloride hexahydrate), diammonium oxalate, $\mathrm{ZnSO}_{4} 7 \mathrm{H}_{2} \mathrm{O}$ (zinc sulphate heptahydrate), $\mathrm{Ni}\left(\mathrm{NO}_{3}\right)_{2} 6 \mathrm{H}_{2} \mathrm{O}$ (nickel nitrite hexahydrate), $\mathrm{NaOH}$, Nutrient agar, Nutrient broth, ethanol and Methanol.

\subsection{Bacterial Strains}

These stains were obtained from PCSIR laboratories Lahore, Pakistan. Escherichia Coli is responsible for urinary tract infections, food poisoning, neonatal meningitis where as Bacillus subtilis, is responsible for sepsis, pneumonia and meningitis in human beings.

Following two methods are employed for the synthesis of Ni-doped $\mathrm{ZnO}$ nano-particles.

\subsection{Method-I}

Nano-particles were synthesized by solvo-thermal method through oxalate precursor route. $0.1 \mathrm{M}$ solutions were prepared by mixing $\left(\mathrm{Zn}(\mathrm{CH} 3 \mathrm{COO})_{2} 2 \mathrm{H}_{2} \mathrm{O}\right) 22.277 \mathrm{~g}$ in $1000 \mathrm{ml} \mathrm{DW}$ and $\mathrm{NiCl}_{2} 6 \mathrm{H}_{2} \mathrm{O} 23.769 \mathrm{~g}$ in $1000 \mathrm{ml} \mathrm{DW}$ water. Stoichiometric amount of zinc acetate dihydrate $(\mathrm{CDH}, 98.5 \%)$ and nickel chloride hexahydrate $\mathrm{NiCl}_{2} 6 \mathrm{H}_{2} \mathrm{O}$ (Merck, 97\%) were stirred for about 30 minutes. The mixture was precipitated with $175 \mathrm{~mL}$ of aqueous solution of diammonium oxalate. $75 \mathrm{ml}$ of ethanol was also added to the reaction mixture. A light green suspension formed. The mixture was refluxed at about $80{ }^{\circ} \mathrm{C}$ for 12 hours. The precipitate was recovered by centrifugation and dried in an oven at $55{ }^{\circ} \mathrm{C}$ and finally ground to powder. Nanoparticles were obtained by thermal decomposition of the precursor at $450{ }^{\circ} \mathrm{C}$ for 6 hours in air.

\subsection{Method-II}

To obtain the desired degree of doping of $\mathrm{Ni}, \mathrm{ZnSO}_{4} 7 \mathrm{H}_{2} \mathrm{O}$ was mixed in distilled water with $\mathrm{Ni}\left(\mathrm{NO}_{3}\right)_{2} 6 \mathrm{H}_{2} \mathrm{O}$. These solutions are designated as solution A. Solution A was placed in an ultrasonic cleaner operating at $57 \mathrm{kHz}$ for 2 h. Solution B is concentrated solution of $\mathrm{NaOH}$ in de-ionized water. After sonication, solution A was stirred with a magnetic stirrer at room temperature. Then solution B was added until a $\mathrm{pH}$ of 12 was reached. The solution was magnetically stirred for $0.5 \mathrm{~h}$, and then the solution was allowed to stand at room temperature for $18 \mathrm{~h}$. Subsequently, the solution was centrifuged and washed several times with ethanol and distilled water to remove residual and unwanted impurities. The final product was dried in a vacuum oven at $200^{\circ} \mathrm{C}$ for $1 \mathrm{~h}$ to yield $\mathrm{Ni}$-doped $\mathrm{ZnO}$ particles. 


\subsection{Characterization}

The structural characterization of synthesized nano-particles was carried out by SEM. For further characterization and average crystallite size determination XRD was also performed. The results were given in Table 1 and 2. Doping of $\mathrm{Ni}$ on $\mathrm{ZnO}$ was confirmed by EDX spectra. UV-VIS-spectrophotometer was used to check photo activity of nano-particles by energy band gap studies.

\subsection{Bacterial Culture Used for Antibacterial Study}

The prepared and sterilized agar solution was poured in sterilized petri plates under laminar and allows cooling up to room temperature. $1 \mathrm{ml}$ of inoculum was added to each plate immediately into medium plates and was shaken gently. The petri plates then allow standing until bacteria cultured media become semisolid. Wells were created by using stainless steel borer, four wells in each petri plate. Negative and Positive controls in two wells were applied separately in each petri plate along with two wells containing different concentrations of sample solutions by using micro syringe. After that these petri plates were placed for 24 hours at $37^{\circ} \mathrm{C}$ in an incubator. After allotted time petri plates were taken out from incubator diameters of inhibition zones were measured using millimeters scale. Each experiment was made in triplicate and the inhibition zones are given as the mean \pm standard deviation. The results were recorded in Table 3 (Leven et al., 1979).

\subsection{Minimum Inhibitory Concentration (MIC)}

For determination of MIC recommended methods of NCCLS; 2000 in modified version was employed. In short, aerobically incubated tubes contained $5 \mathrm{~mL}$ Muller- Hinton (MH) broth (Difco, USA) with approximate $5 \times 10^{9}$ CFU bacterial cells for 24 hours at $37^{\circ} \mathrm{C}$, without nano-particles (the control group) and various concentrations of synthesized nano-particles. The MIC is determined from the tube without visible growth of the bacterial cells.

\subsection{Minimum Bactericidal Concentration (MBC)}

In order to find $\mathrm{MBC} 100 \mu \mathrm{L}$ of sample from the tube without visible growth transferred into $\mathrm{MH}$ agar plate (Difco, USA), and it was then incubated aerobically again for another $24 \mathrm{~h}$ (NCCLS, 2000). The concentration of tube without growth was the MBC.

\section{FINDINGS AND DISCUSSIONS}

\subsection{XRD Analysis}

XRD diffraction patterns of $\mathrm{Ni}$-doped zinc oxide nano-particles synthesized through co-precipitation method are shown in figure-1 and the XRD diffraction patterns of Ni-doped zinc oxide nano-particles form through solvo-thermal method through oxalate precursor route are shown in figure-2. The nano-particles obtained through co-precipitation method are termed as $M_{1}$. On the other hand nano-particles obtained through solvo-thermal method are termed as $M_{2}$.

Fig 1: X-ray pattern of the Ni doped ZnO nano-particles $\mathrm{M}_{1}$

Fig 2: X-ray pattern of the Ni doped ZnO nano-particles $\mathrm{M}_{2}$
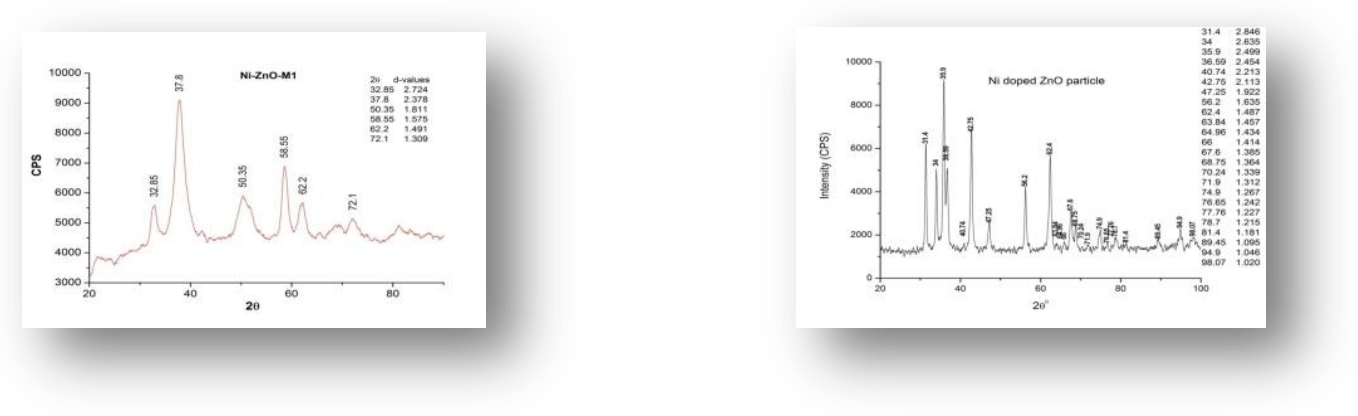

The obtained XRD diffraction patterns having the peaks match to the characteristic peaks of ZnO XRD diffraction pattern (JCPDS file No: 36-1451). Similarly the XRD diffraction patterns of Ni-doped ZnO also match to the characteristic peaks $\mathrm{Ni}$ doped ZnO XRD diffraction pattern (JCPDS card no.78-0643) confirming the doping and synthesis of desired product along with absence of any impurity present in synthesized samples.

\subsection{Crystalite Average Size of Nano-Particles}

The Crystallite average sizes of all prepared samples of $\mathrm{Ni}$-doped zinc oxide nano-particles were calculated for all the using Scherrer formula. 


$$
D=\frac{\mathrm{K} \lambda}{\beta \cos \theta}
$$

Here $\mathrm{K}$ is constant estimated as 0.9 , peak width is represented by $\beta$ which is calculated at half height of peak in radians, $\lambda$ represents wavelength of radiation used. Angle of reflection is shown " $\theta$ ".

Table 1: Crystallite Size of Ni Doped ZnO Nano-Particles $M_{1}$

\begin{tabular}{ccccc}
\hline $\begin{array}{c}2 \theta \text { (deg) of the } \\
\text { Intense peak }\end{array}$ & $\begin{array}{c}\text { FWMH } \\
\text { Intense peak } \\
\left(\theta_{2}-\theta_{1}\right)\end{array}$ & $\begin{array}{c}\text { FWMH } \\
\text { Intense peak }(\beta) \\
\text { radians }\end{array}$ & $\begin{array}{c}\text { Grain Size } \\
\text { (D) } \\
\mathrm{nm}\end{array}$ & $\begin{array}{c}\text { Average Grain Size } \\
\mathrm{nm}\end{array}$ \\
\hline 37.8 & 1.99 & 0.035 & 4.17 & \\
58.5 & 1.26 & 0.022 & 7.20 & $6.32 \mathrm{~nm}$ \\
32.8 & 1.09 & 0.019 & 7.59 & \\
\hline
\end{tabular}

Table 2: Crystallite Size of Ni Doped ZnO Nano-Particles $M_{2}$

\begin{tabular}{ccccc}
\hline $\begin{array}{c}2 \theta \text { (deg) of the } \\
\text { Intense peak }\end{array}$ & $\begin{array}{c}\text { FWMH } \\
\text { Intense peak } \\
\left(\theta_{2}-\theta_{1}\right)\end{array}$ & $\begin{array}{c}\text { FWMH } \\
\text { Intense peak }(\beta) \\
\text { radians }\end{array}$ & $\begin{array}{c}\text { Grain Size } \\
(D) \\
\mathrm{nm}\end{array}$ & $\begin{array}{c}\text { Average Grain Size } \\
\mathrm{nm}\end{array}$ \\
\hline 36.2 & 1.63 & 0.028 & 5.19 & \\
42.7 & 1.09 & 0.019 & 7.81 & $7.17 \mathrm{~nm}$ \\
62.4 & 1.09 & 0.019 & 8.51 & \\
\hline
\end{tabular}

The average crystallite sizes for sample $M_{1}$ is calculated as $6.32 \mathrm{~nm}$ and for sample $M_{2}$ is $7.17 \mathrm{~nm}$. This crystallite size is also called particle diameter. From these results it is clear that all the synthesized samples from both the methods having size in nano-scale range.

\subsection{SEM Results}

The SEM results for all the samples are shown above give detailed view about the crystal structure, particle size and morphology. The SEM results shows that the particles produced through co-precipitation method are of high density, their morphology and shape of crystals shown in figure 3. Nano-particls produced through solvo-thermal method shown in figure 4. Although doping of $\mathrm{Ni}$ over $\mathrm{ZnO}$ and formation of nano-particles were confirmed by XRD but the samples were not further considered for Energy Dispersive X-ray (EDX) analysis due to SEM results. However samples were considered for optical activity through band gap analysis. The SEM results for particles produced through solvo-thermal were found better in crystal structure and morphology.

\section{Figure 3: SEM of $M_{1}$}

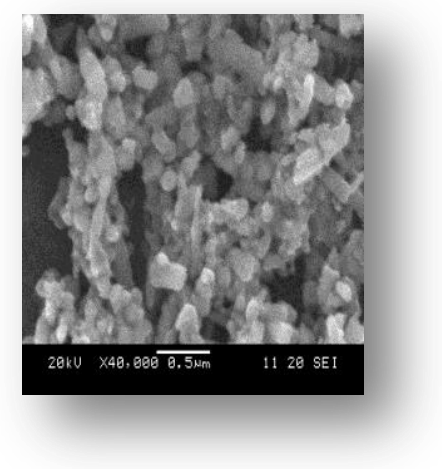

Figure 4: SEM of $\mathrm{M}_{2}$

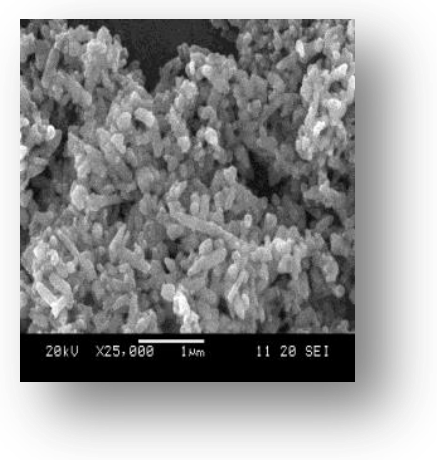

\subsection{EDX Analysis}

The extant of Ni doping in Ni-doped zinc oxide samples was checked using Energy Dispersive X-ray analysis. For this purpose $\mathrm{M}_{2}$ (Ni-doped $\mathrm{ZnO}$ sample) produced from solvo-thermal method is used. The area taken for EDX analysis is given below in figure. 


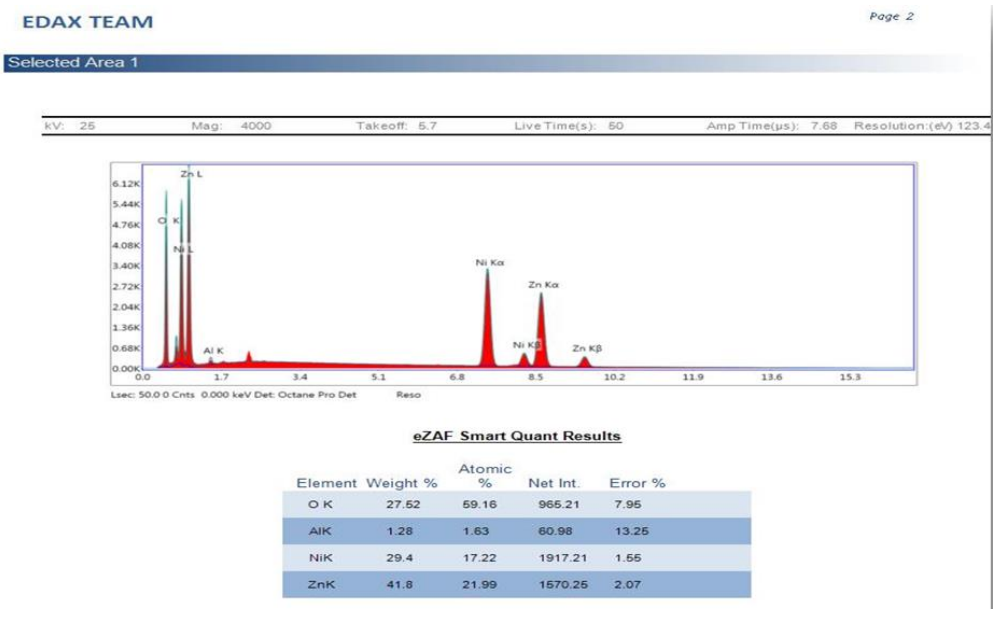

Figure $5 \mathrm{EDX}$ spectra of $\mathrm{Ni}$-doped $\mathrm{ZnO}$ nano-particles synthesized by solvo-thermal method. EDX results shown above were confirmed Ni doping in $\mathrm{ZnO}$ material. The element and weight percentage compositions of $\mathrm{Ni}, \mathrm{Zn}$ and $\mathrm{O}$ in the synthesized nano-particles are given in above table. As a result, the accumulation of $\mathrm{Ni}$ in the $\mathrm{ZnO}$ system dominantly affects the optical, morphological and structural properties of $\mathrm{ZnO}$.

\subsection{Band Gap Analysis}

Ultra Violet visible spectroscopy is used to study optical activity of Ni-doped zinc oxide nano-particles. Band gap results using Ultra Violet visible spectroscopy were obtained. UV-VIS band gap results of $M_{1}$ and $M_{2}$ are shown in Figures below.

Figure 6: Band Gap for $M_{1}$

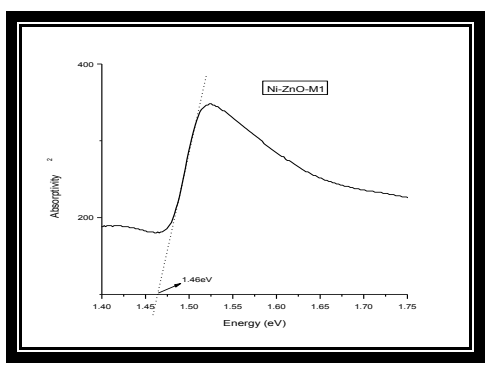

Figure 7: Band Gap for $\mathrm{M}_{2}$

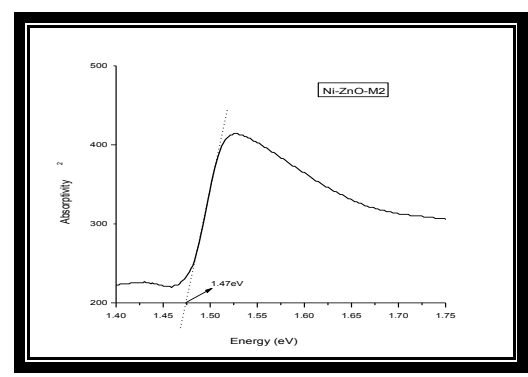

The optical band gaps are calculated by using Tauc equation as given below:

$$
\alpha=\frac{C\left(h \vartheta-E_{g}^{\text {bulk }}\right)^{2}}{h \vartheta}
$$

Here $\alpha$ is absorption coefficient, $C$ is constant, hu is photon energy and $\mathrm{E}_{\mathrm{g}}{ }^{\text {bulk }}$ is band gap, respectively.

Samples which synthesized by both the methods have been successfully studied using UV-Vis. All samples have shown optical activity which is indicated by the band gap values. Sample produced through solvo-thermal method showed slight higher band gap values than the sample produced through co-precipitation method. As a whole we can conclude that $\mathrm{Ni}$ doping enhances the optical activity of $\mathrm{ZnO}$ system or we can say Ni-doped zinc oxide nano-particles shown higher optical activity than un-doped $\mathrm{ZnO}$ nano-particles.

\subsection{Antibacterial Activity Results}

After the specified time for incubation the petri plates under test were taken out from the incubator and zones of inhibition were observed under Laminar flow hood. Clear zones of inhibition were observed. Antibacterial activity of synthesized Nidoped zinc oxide nano-particles was dose dependent. It was also clear form antibacterial activity experimental results that antibacterial activity was directly proportional to concentration. The obtained data also showed bacterial sensitivity of $\mathrm{Ni}-$ doped zinc oxide nano-particles against two different bacterial classes. The bacterial sensitivity is more against Gram positive bacteria with comparison of Gram negative bacteria for all synthesized samples. Ni-doped zinc oxide nano-particles were stable and their potent antibacterial properties favors them to be used as antibacterial agent against broad spectrum microorganisms. 
Table 3: Zones of Inhibitions of Ni-Doped ZnO Nano-Particles against Different Bacterial Strains

\begin{tabular}{cccc}
\hline \multirow{2}{*}{ Bioactive Agent } & \multicolumn{2}{c}{ Zone of Inhibition* (Diameter, mm) } \\
\cline { 3 - 4 } & $100 \mu \mathrm{L}$ & Escherichia Coli & Bacillus subtilis \\
\cline { 3 - 4 } Ni doped ZnO & $200 \mu \mathrm{L}$ & $11 \pm 1.2$ & $14 \pm 0.3$ \\
\cline { 3 - 4 } nano-particles & $300 \mu \mathrm{L}$ & $13 \pm 0.9$ & $16 \pm 0.2$ \\
\cline { 3 - 4 } & $400 \mu \mathrm{L}$ & $14 \pm 0.1$ & $18 \pm 0.5$ \\
\cline { 3 - 4 } & $500 \mu \mathrm{L}$ & $15 \pm 0.8$ & $20 \pm 0.6$ \\
\cline { 3 - 4 } $\begin{array}{c}\text { Cephradinen } \\
\text { Ampicillin } \\
\text { trihydrate }\end{array}$ & $100 \mu \mathrm{L}$ & $16 \pm 0.6$ & $23 \pm 1.2$ \\
\hline
\end{tabular}

*Inhibition zone $(\mathrm{mm})$ include the diffusion assay disc diameter $(4 \mathrm{~mm})$, which carried $50 \mu \mathrm{L}$ from nano-particles suspension. The diameter of inhibition zones are means triplicate \pm standard deviation. $p<0.05$ when compared with negative control i.e. blank/solvent ( $p<0.05$ is taken as significant).

The MIC is the concentration at which the solution becomes turbid. Small value of MIC for to a material depicts its higher antibacterial effectiveness.

\section{CONCLUSION}

Ni-doped zinc oxide nano-particles were successfully synthesized by co-precipitation method and solvo-thermal methods. Characterizations of prepared samples were performed using XRD analysis. Particles were found crystalline in nature and their particle size was also found in nanometers. Extant of Ni-doping over zinc oxide was calculated using EDX analysis. The EDX analysis confirmed successful preparation of Ni-doped zinc oxide nano-particles. Furthermore, SEM analysis un-doped and $\mathrm{Ni}$-doped zinc oxide nano-particles were performed which showed that well shaped geometrical nano-particles were prepared from both methods. The optical activity of synthesized samples was checked, band gaps were calculated for using UV-VIS spectroscopy. Band gap results showed that doping of zinc oxide nano-particles with $\mathrm{Ni}$ enhances their optical activity. Antibacterial activity for all synthesized samples was checked using ager well diffusion method. The samples had worked as effective antibacterial agents. Their activity was found more promising against Gram (+) bacteria as compared with Gram (-) bacteria. MIC for the synthesized nano-particles was proving them as future antibacterial agent. The synthesized nano-particles can be used as potential candidate for solar cells aplications. Moreover their use in nanocomposite materials may also be investigated in future.

\section{REFERENCES}

Al-Harbi, T. (2011). Hydrothermal synthesis and optical properties of Ni doped ZnO hexagonal nanodiscs. J. Alloy. Compd. 509(2), 387-390.

Ananda, S. (2014). Synthesis and Characterization of Se-doped ZnO Nanoparticles by Electrochemical Method: Photodegradation Kinetics of Indigo Carmine Dye and Study of Antimicrobial, Antimitotic Activities of Se-doped ZnO Nanoparticles. J. Am. Chem. Sci. 4(5), 616-637.

Buzea, C., Pacheco, I. I., \& Robbie, K. (2007). Nanomaterials and nanoparticles: sources and toxicity. Biointerphases, 2(4), MR17-MR71.

Cai, A. J., Wang, Y. L., Xing, S. T., Du, L. Q., \& Ma, Z. C. (2013). Tuned morphologies of DNA-assisted ZnO struggling against pH. Ceram. Int. 39(1), 605-609.

Hewakuruppu, Y. L., Dombrovsky, L. A., Chen, C., Timchenko, V., Jiang, X., Baek, S., \& Taylor, R. A. (2013). Plasmonic "pump-probe" method to study semi-transparent nanofluids. Appl. Opt., 52(24), 6041-6050.

Hua, G., Zhang, Y., Ye, C., Wang, M., \& Zhang, L. (2007). Controllable growth of ZnO nanoarrays in aqueous solution and their optical properties.Nanotechnology, 18(14), 145605.

Hsu, H. C., Cheng, H. M., Wu, C. Y., Huang, H. S., Lee, Y. C., \& Hsieh, W. F. (2006). Luminescence of selective area growth of epitaxial ZnO nanowires and random-growth-oriented nanobelts. Nanotechnology, 17(5), 1404.

Khatoon, S., \& Ahmad, T. (2012). Synthesis, optical and magnetic properties of Ni-doped ZnO nanoparticles. J. Mat. Sci. Engine. B, 2(6), 325-333.

Leven, M., Berghe, D. A. V., Mertens, F., Vlietinck, A., \& Lammens, E. (1979). Screening of higher plants for biological activities I. Antimicrobial activity. Planta Medica, 36(08), 311-321.

Liewhiran, C., Seraphin, S., \& Phanichphant, S. (2006). Synthesis of nano-sized ZnO powders by thermal decomposition of zinc acetate using Broussonetia papyrifera (L.) Vent pulp as a dispersant. Curr. Appl. Phy. 6(3), 499-502. 
Moriga, T., Y. Hayashi, Y,. Kondo, K., Nishimura, Y. (2004). Zinc oxide, tin oxide-based transparent conductive thin film of amorphous, J. Vac. Sci. and Technol A, 22, 705-1710.

Raja, K., Ramesh, P. S., \& Geetha, D. (2014). Synthesis, structural and optical properties of ZnO and Ni-doped ZnO hexagonal nanorods by Co-precipitation method. Spectrochimica Acta Part A. 120, 19-24.

Sahoo, S. K., Parveen, S., \& Panda, J. J. (2007). Clinical nanomedicine. The present and future of nanotechnology in human health care. Nanomed. Nanotechnol. Biol. Med. 3(1), 20-31.

Siegel, R. W. (1994). What do we really know about the atomic-scale structures of nanophase materials?. J. Phy. Chem. Solids, 55(10), 1097-1106.

Wang, Z. L. (2004). Zinc oxide nanostructures: growth, properties and applications. J. Physics: Condensed Matter, 16(25), R829.

Xu, M., Fujita, D., Kajiwara, S., Minowa, T., Li, X., Takemura, T., ... \& Hanagata, N. (2010). Contribution of physicochemical characteristics of nano-oxides to cytotoxicity. Biomaterials, 31(31), 8022-8031.

Yan, H., Johnson, J., Law, M., He, R., Knutsen, K., McKinney, J. R., ... \& Yang, P. (2003). ZnO nanoribbon microcavity lasers. Adv. Mat. 15(22), 1907-1911.

Zhang, J., Wang, S., Xu, M., Wang, Y., Zhu, B., Zhang, S., ... \& Wu, S. (2009). Hierarchically porous ZnO architectures for gas sensor application. Crys. Gro. Degn., 9(8), 3532-3537.

Zhong, Q., \& Matijević, E. (1996). Preparation of uniform zinc oxide colloids by controlled double-jet precipitation. J. Mat. Chem. 6(3), 443447. 